\title{
A tidal disruption model for the gamma-ray burst of GRB 060614
}

\author{
Y. Lu ${ }^{1}$, Y.F. Huang ${ }^{2}$, and S.N. Zhang ${ }^{3}$
}

\begin{abstract}
The combination of a long duration and the absence of any accompanying supernova clearly shows that GRB 060614 can not be grouped into the two conventional classes of gamma-ray bursts, i.e. the long/soft bursts deemed to be collapsars and the short/hard bursts deemed to be merging binary compact stars. A new progenitor model is required for this anomalous gamma-ray burst. We propose that GRB 060614 might be produced through the tidal disruption of a star by an intermediate mass black hole. In this scenario, the long duration and the lack of any associated supernova are naturally expected. The theoretical energy output is also consistent with observations. The observed 9-s periodicity in the $\gamma$-ray light curve of GRB 060614 can also be satisfactorily explained.
\end{abstract}

Subject headings: gamma rays: bursts - black hole physics

\section{Introduction}

Gamma-ray bursts (GRBs) are generally grouped into two classes, namely long/soft bursts and short/hard bursts, separated at $T_{90} \sim 2 s$ (Kouveliotou et al. 1993). Long GRBs are believed to originate from the collapse of massive stars (Woosley 1993). They are usually associated with supernovae (Galama et al. 1998; Pian et al. 2006), and occur in star forming regions (Fruchter et al. 2006). Short GRBs are widely believed to be connected with the merge of binary compact stars, and their host galaxies are thought to have a lower star formation rate (Blinnikov et al. 1984; Eichler et al. 1989; Villasenor et al. 2005; Bloom et al. 2006). They should not be associated with any supernovae. Recently, by analogy with the SN classification, Zhang et al. (2007) suggested a new GRB terminology scheme: Type I and

\footnotetext{
${ }^{1}$ National Astronomical Observatories, Chinese Academy Of Sciences, Beijing 100012, China; ly@bao.ac.cn

${ }^{2}$ Department of Astronomy, Nanjing University, Nanjing 210093, China

${ }^{3}$ Physics Department and Center for Astrophysics, Tsinghua University, Beijing 100084, China
} 
Type II bursts. Type I bursts correspond to the star merging group, and Type II bursts correspond to the collapse of massive stars.

GRB 060614 is a very special event that has strongly challenged our classical classification of GRBs (Gehrels et al. 2006). It has a long duration of $\sim 102 \mathrm{~s}$ (Gehrels et al. 2006), which seems to suggest it as a long/soft GRB. However, no supernova signature has been observed from this event, although it is very near to us (Gal-Yam et al. 2006; Fynbo et al. 2006), which is strongly contradicted with a collapsar origin. Obviously, this GRB cannot be simply classified as any of the conventional class, either long/soft GRBs or short/hard GRBs. A novel mechanism is needed for this special burst (Gal-Yam et al. 2006; Zhang et al. 2007; Mangano et al. 2007; Jakobsson \& Fynbo 2007; Amati et al. 2007).

Although the formation mechanism of intermediate mass black holes (IMBHs) is still not clear so far (Heger \& Woosley 2002; Portegies et al. 2004; Miller \& Hamilton 2002; Gebhardt et al. 2005), some evidence has been accumulated for their existence in galaxies of all types, including dwarf galaxies, especially in young and global clusters (Mapelli 2007). We propose here that the tidal disruption of a star by an IMBH could be an ideal way to power a nearby long-duration GRB without any associated supernova, such as GRB 060614. Our motivation is that the debris of the tidally disrupted star is likely to form an accretion disk surrounding the black hole. As a consequence, jets would be formed in this process (Lu et al. 2006), which are similar to the jets triggered by collapsars and binary mergers. We believe that the debris accreted onto the IMBH is a conceivable energy source for GRBs.

The structure of this paper is as follows. We gather up the observed facts of GRB 060614 in Section 2. Our novel model for GRB 060614 is described in detail in Section 3. Section 4 is the conclusion and a brief discussion.

\section{Main features of GRB 060614}

GRB 060614 is a very peculiar nearby burst, which was detected by the Burst Alert Telescope (BAT) onboard the Swift satellite on 2006 June 14 at 12:43:48 UT (Gehrels et al. 2006). The general properties of GRB 060614 can be summarized as follows. (1) It is a long GRB that lasted for $\sim 102 \mathrm{~s}$ (Gehrels et al. 2006); (2) An interesting substructure has been noted in the prompt $\gamma$-ray light curve: BAT records reveal a first short episode of emission lasting for $\sim 4 \mathrm{~s}$ followed by an extended and somewhat softer episode lasting for $\sim 100 \mathrm{~s}$ (Gehrels et al. 2006; Fynbo et al. 2006). A close examination shows that this short episode is actually composed of $\sim 5$ mini-pulses. It is also noted that a 9-s periodicity exists between 7 and $50 \mathrm{~s}$ in the $\gamma$-ray light curve, although it is not statistically significant (Gehrels et al. 
2006). (3) It has a long duration, but it lies in the region of short/hard GRBs on the temporal lag-peak luminosity plane (Gehrels et al. 2006); (4) It is not associated with any supernova, although it is at a relatively low redshift of $z=0.125$ (Gal-Yam et al. 2006; Fynbo et al. 2006); (5) Its host is a faint dwarf galaxy, with a low star formation rate of $0.0084-0.014$ $\mathrm{M}_{\odot} /$ yr (Jakobsson \& Fynbo 2007; Gal-Yam et al. 2006; Fvnbo et al. 2006); (6) The GRB is offset from the nucleus of the host galaxy by 0.5" (Gehrels et al. 2006; Gal-Yam et al. 2006); (7) The gamma-ray fluence is $\sim 2 \times 10^{-5} \mathrm{ergs} / \mathrm{cm}^{2}$, which corresponds to an isotropic gamma-ray energy release of $1.08 \times 10^{51} \mathrm{ergs}$ in $1 \mathrm{keV}-10 \mathrm{MeV}$ range in the GRB restframe (Gehrels et al. 2006). Note that if the radiation efficiency is 0.1 , then the isotropic kinetic energy is $\sim 1 \times 10^{52}$ ergs.

The long duration of GRB 060614 suggests it as a long/soft GRB. However, the absence of any associated supernova signature of such a nearby event strongly contradicts a collapsar origin. The above observational facts indicate that GRB 060614 does not fit into any of the two conventional classes of GRBs, i.e. long/soft and short/hard GRBs. A completely new type of engine other than the collapsars or binary compact star mergers is need for this event. Below, we will show that the tidal disruption of a star by an IMBH can hopefully explain all the basic features of GRB 060614.

\section{Tidal disruption model for GRB 060614}

There are three kinds of black holes: stellar mass black holes, intermediate mass black holes (IMBHs), and super-massive black holes. Studies have been abundant for stellar mass black holes and super-massive black holes. Stellar mass black holes, with masses ranging from 3 to $20 \mathrm{M}_{\odot}$ (Orosz 2003), are thought to be the relics of massive stars. Super-massive black holes, with a mass of $M_{\mathrm{bh}} \sim 10^{6}-10^{9} \mathrm{M}_{\odot}$, are believed to reside at the center of many galaxies. Strong observational evidence for the existence of stellar mass black holes and super-massive black holes has been accumulated today. For example, tidal disruption of a star by a super-massive black hole is expected to produce a luminous flare of electromagnetic radiation in the UV to X-ray bands at the center of many galaxies (Rees 1988; Komossa \& Greiner 1999; Ulmer 1999; Komossa et al. 2004; Milosavljević et al. 2006). This process is also suggested as a possible source for the observed non-thermal TeV gamma-ray emission from the center region of our Galaxy ( $\mathrm{Lu}$ et al. 2006). Interestingly, as one potential model for long GRBs (Trimble \& Aschwanden 2001), the tidal disruption of a star by a super-massive black hole has already been discussed in the earlier works of Carter (1992) and Cheng \& Lu (2001). Furthermore, a possibility of gamma-ray flares linked to shock waves in tidally compressed stars by massive black holes has been predicted in the more recent 
work of Brassart \& Luminet (2007).

The masses of IMBHs are in the range of $M_{\mathrm{bh}} \sim 20-10^{5} \mathrm{M}_{\odot}$ (Mapelli et al. 2006). Their existence has also been inferred from recent observations. For example, the capture of stars by IMBHs with masses of $350-1200 \mathrm{M}_{\odot}$ are suggested as the mechanism for some ultraluminous X-ray sources (Baumgardt et al. 2006). In this section, we investigate the possibility of producing a GRB 060614-like GRB through the tidal disruption of a star by an IMBH. The main difference between this GRB model and the former one proposed by Cheng \& Lu (2001) is the mass of black holes and the location in the host galaxies. Note that IMBHs can be offset from the center region of their host galaxy.

\subsection{General picture}

Despite the great difference between the black hole masses, tidal disruption of a star by an IMBH should be more or less similar to the disruption of a star by a super-massive black hole. A transient accretion disk may be formed in the process. The accretion rate should be very high (beyond the Eddington rate) at first, but should decrease steadily with time. As long as the accretion rate is high enough (nearly the Eddington rate), the inner region of the disk should be dominated by radiation pressure. The disk can then anchor and amplify the seed magnetic field $\left(B_{\text {eq }}\right)$ to a strong ordered poloidal field $\left(B_{\mathrm{p}}\right)$, which in turn threads the black hole with a mass-flow ring in the inner region of the disk. A large amount of rotational energy of the black hole can be extracted via the Blandford-Znajek (BZ) process, creating two counter-moving jets along the rotation axis (Blandford \& Znajek 1977). The powerful jets are ideal energy reservoirs for GRBs.

A jet produced in such a way should be highly variable. The exponential increase of $B_{\text {eq }}$ to $B_{\mathrm{p}}$ is linked to an instability of the disk in the case of a high accretion rate. When $B_{\mathrm{p}}$ is so strong that it dominates over the material pressure, all debris material within the spherization radius $\left(R_{\mathrm{sp}}\right)$ (Shakura \& Sunyaev 1973) is likely to be broken into blobs and fall successively into the black hole at the marginally stable radius $\left(R_{\mathrm{ms}}\right)$. The falling of each blobs into the black hole should lead to a clump-like structure in the jet, which may correspond to a pulse (a mini-burst) in the light curve of the GRB (Cheng \& Lu 2001). The width of a mini-burst $\left(t_{\text {pulse }}\right)$ is determined by the free-fall timescale $\left(t_{\mathrm{ff}}\right)$. The total duration of the whole GRB ( $\left.t_{\text {duration }}\right)$ is determined by the time needed for all the debris material in the range of $R_{\mathrm{ms}}<R<R_{\mathrm{sp}}$ to fall into the black hole at $R_{\mathrm{ms}}$, which in fact is the instability timescale of the disk $\left(t_{\text {in }}\right)$.

The instability should happen when the accretion rate is near the Eddington rate. Note 
that when all the original material in the range of $R_{\mathrm{ms}}<R<R_{\mathrm{sp}}$ is accreted by the black hole, the region cannot be replenished again, due to the combination of the relatively long accretion timescale and the rapidly decreasing accretion rate. The central engine is then actually quenched, although consequent energy injection is still possible due to continuous accretion at a much lower level.

\subsection{Tidal disruption and the formation of a transient accretion disk}

When a star with a mass of $M_{*}$ and radius of $R_{*}$ passes by a massive black hole with a mass of $M_{\mathrm{bh}}$, it would be captured and eventually tidally disrupted at an average tidal radius of

$$
R_{\mathrm{T}} \simeq 3.25 \times 10^{12} r_{*} m_{*}^{-1 / 3} M_{5}^{1 / 3} \mathrm{~cm}
$$

where $m_{*}=M_{*} / M_{\odot}, r_{*}=R_{*} / R_{\odot}, R_{\odot}$ and $M_{\odot}$ are the solar radius and mass, respectively. For convenience, we introduce the following dimensionless quantities throughout this article:

$$
M_{5}=\frac{M_{\mathrm{bh}}}{10^{5} M_{\odot}}, \dot{m}=\frac{\dot{M}}{\eta \dot{M}_{\mathrm{Edd}}}, \hat{r}=\frac{R}{R_{\mathrm{ms}}},
$$

where $R_{\mathrm{ms}}=3 R_{\mathrm{s}}$ and $R_{\mathrm{s}}=3 \times 10^{10} M_{5} \mathrm{~cm}$ is the Schwarzschild radius, $\dot{M}_{\mathrm{Edd}}=3 \times$ $10^{-2} \eta_{0.1}^{-1} M_{5} M_{\odot} / \mathrm{yr}$ is the Eddington accretion rate, $\eta$ is the energy conversion factor and $\eta_{0.1}=\eta / 0.1$.

The strength of a tidal disruption generally depends on the black hole mass and the penetration factor $\beta$ defined as $\beta \equiv R_{\mathrm{T}} / R_{\mathrm{p}}$, where $R_{\mathrm{p}}$ is the pericenter of the star's orbit. The maximum value of $\beta$ is 12 and 56 for $M_{\mathrm{bh}}=10^{6}$ and $10^{5} \mathrm{M}_{\odot}$. Schwarzschild black hole (Kobayashi et al. 2004), respectively. When $\beta \gg 1$, the star within $R_{\mathrm{T}}$ can undergo compression to a highly flattened pancake configuration (Carter \& Luminet 1983; Luminet \& Carter 1986; Luminet \& Pichon 1989). Once the star is totally disrupted in such a way, the matter with higher angular momentum will rapidly lag behind the matter with lower angular momentum, producing a long and thin spiral (Gomboc \& C̆adež 2005), and resulting in a continuous debris accreted onto the black hole. Although it is difficult to predict what fraction of the initially bound debris will be accreted, the recent numerical simulations indicate that approximately $25 \%$ - 50\% of the initial stellar mass may remain bound (Aval et al. 2000). At first the accretion rate may be much higher than the Eddington rate and no accretion disk with a radiation-dominated inner region can be formed. We will not discuss this phase since no powerful jet can be launched during this phase. When the accretion rate decreases to about the Eddingtion rate, a disk of our interest may be formed. The radius of 
the disk is typically comparable to the tidal radius $R_{\mathrm{T}}$, and the evolution of the accretion rate is given by (Rees 1988)

$$
\dot{m}=2.64 \times 10^{3}\left(\frac{t}{t_{\mathrm{D}}}\right)^{-5 / 3},
$$

where $t_{\mathrm{D}}$ is the dynamic or orbit timescale, which is given by (Sanders \& Van Oosterom 1984)

$$
t_{\mathrm{D}}=\left(\frac{G M_{\mathrm{bh}}}{R_{\mathrm{T}}^{3}}\right)^{-1 / 2}=1.6 \times 10^{3} r_{*}^{3 / 2} m_{*}^{-1 / 2} \mathrm{~s} .
$$

Assuming that the evolution of the debris disk follows the behavior of a standard disk, then the infall of matter within a narrow sector (a mass ring) is a special kind of disk accretion. The matter moves in a slowly twisting spiral in the plane perpendicular to the direction of the angular momentum of the mass flow. The time in which a large fraction of the matter in the disk can be accreted is (Ulmer 1999)

$$
t_{\mathrm{acc}} \approx 9.47 \times 10^{7} \alpha^{-1} h_{-2}^{-2} \beta^{-3 / 2} r_{*}^{3 / 2} m_{*}^{-1 / 2} s,
$$

where $\alpha$ is the viscous paramater, ranging as $0 \leq \alpha \leq 1, h=10^{-2} h_{-2}=H / R$ is the ratio of disk height $(H)$ to radius and is approximately one for a thick disk. For a thin disk, we adopt $h=10^{-2}$. In this case, the accretion time is very long. It indicates that when a region of the disk is suddenly cleaned up due to some mechanism, it would essentially be impossible to replenish this region again, due to the relatively long accretion timescale $\left(t_{\text {acc }}\right)$ and the rapidly decreasing accretion rate.

There are three distinct regions in a standard thin disk, depending on the sources of opacity and pressure (Shakura \& Sunyaev 1973): the inner region, the middle region and the outer region. We concentrate on the inner region, which is dominated by radiation pressure and electron scattering. Note that this region exists only when the accretion rate is high, i.e. $\dot{m}>\dot{m}_{\mathrm{c}}$, where $\dot{m}_{\mathrm{c}}=7.97 \times 10^{-3}\left(\alpha M_{5}\right)^{-1 / 8}$ is a critical accretion rate (Shakura \& Sunvaev 1973). In this case, the disk has a constant thickness along the radius for $\hat{r} \gg 1$, and the thickness depends only on the accretion rate. The maximal value of $h$ is reached at $R_{\mathrm{sp}}$ as $h_{\max }=\dot{m}$, where $R_{\mathrm{sp}}$ is the radius of spherization (Shakura \& Sunyaev 1973)

$$
R_{\mathrm{sp}}=2.25 \times 10^{11} \dot{\mathrm{m}} M_{5} \mathrm{~cm} .
$$

Within the radius of $R_{\mathrm{sp}}$, the disk may suffer an instability, e.g., due to mass accumulation resulted from the increase of the viscosity and the local accretion rate. A thermal ionization instability is eventually developed. Consequently, magnetic field can be amplified in the process and jets can be launched via the BZ mechanism. 


\subsection{Energy extraction}

To estimate the powerful energy extracted via BZ process, we need to calculate the ordered poloidal field. Irrespective of the detailed field structure, the original magnetic field in the disk can be estimated by the assumption of energy equipartition: $B_{\text {eq }}^{2} /(8 \pi) \equiv p_{\mathrm{d}, \max }$, where $p_{\mathrm{d} \text {,max }}$ is the maximum pressure of the disk. For a high accretion rate, the disk is dominated by the radiation pressure and the maximum pressure in the inner region can be described following the work of Shakura \& Sunyaev (1973),

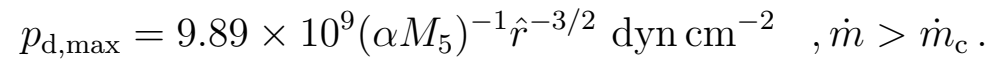

Eq. (5) shows that the maximum pressure is a function of $\hat{r}^{-3 / 2}$. Since $B_{\text {eq }} \propto p_{\mathrm{d} \text {,max }}^{1 / 2}$, we have $B_{\text {eq }} \propto \hat{r}^{-3 / 4}$. $B_{\text {eq }}$ acts as a seed magnetic field. So the seed field is a function of radius. It increases quickly with the decrease of the radius.

The instability happens in the region of $R_{\mathrm{ms}}<R<R_{\mathrm{sp}}$ (Shakura \& Sunyaev 1973; Cheng \& Lu 2001; Lu et al. 2006). We refer this region as the instability region, and designate the corresponding radius as $R_{\text {in }}$. Accordingly the dimensionless radius is $\hat{r}_{\mathrm{ms}}<\hat{r}_{\mathrm{in}}<\hat{r}_{\mathrm{sp}}$, where $\hat{r}_{\mathrm{in}}=R_{\mathrm{in}} / R_{\mathrm{ms}}$, and $\hat{r}_{\mathrm{sp}}=R_{\mathrm{sp}} / R_{\mathrm{ms}}=2.52 \dot{m}$. In this region, the debris matter is likely to be broken into many blobs, and the seed field threading the block and the disk may be wrapped up tightly, becoming highly sheared and predominantly azimuthal in orientation. This leads to a bursting growth of $B_{\text {eq }}$ to a strong poloidal magnetic fields $B_{\mathrm{p}}$ (Cheng \& Lu 2001). The final amplified strength, $B_{\mathrm{p}}\left(\propto B_{\text {eq }}\right)$, and the corresponding growth timescale $\left(\triangle t_{\mathrm{p}}\right)$ are, respectively (Cheng \& Lu 2001),

$$
\begin{aligned}
B_{\mathrm{p}}^{2} & \simeq 5.67 \times 10^{-2} n \mathrm{G}^{2} \\
\triangle t_{\mathrm{p}} & \simeq 4.5 \times 10^{-4} n^{1 / 8} \alpha^{1 / 8} M_{5}^{9 / 8} \hat{r}_{\mathrm{in}}^{3 / 8} \mathrm{~s}
\end{aligned}
$$

where $n$ (in units of $\mathrm{cm}^{-3}$ ) is the number density in the inner region. It is given by (Shakura \& Sunyaev 1973)

$$
n=2.79 \times 10^{21}\left(\alpha M_{5}\right)^{-1} \hat{r}_{\mathrm{in}}^{-9 / 8} \quad\left(\dot{m}>\dot{m}_{\mathrm{c}}\right) .
$$

Substituting Eq.(7) into Eq.(6), we have

$$
\begin{aligned}
& B_{\mathrm{p}}^{2}=1.58 \times 10^{20}\left(\alpha M_{5}\right)^{-1} \hat{r}_{\mathrm{in}}^{-9 / 8} \mathrm{G}^{2} \\
& \triangle t_{\mathrm{p}} \simeq 2.15 \times 10^{-1} M_{5} \hat{r}_{\mathrm{in}}^{15 / 64} \mathrm{~s} .
\end{aligned}
$$

In the region of $R_{\mathrm{ms}}<R<R_{\mathrm{sp}}$, the debris matter is broken into many blobs. The characteristic dimension for each blob is limited by the geometrical thickness of the accretion 
flow, and thus can be estimated as $\sim H$ (Abramowicz 1985). Owing to general relativistic effects, near the black hole, the relative value of $H$ can be estimated by (Abramowicz 1985)

$$
\frac{H}{R_{\text {in }}} \sim 10^{-2} \zeta^{-1 / 2} \chi
$$

where $\chi$ is a parameter depending on the mass of the black hole and the accretion rate, and $\zeta$ is the ratio of the gas pressure to the total pressure of the disk. For massive black holes, $\chi \sim 0.1$, and for a thin disk the value of $\zeta$ ranges as $10^{-4} \leq \zeta \leq 1$. Here, we adopt $\zeta \sim 10^{-3}$ for the disk dominated by radiation pressure. Since the instability region is within $R_{\mathrm{sp}}$, the total number of the blobs appearing in the instability region can be estimated as

$$
N_{\text {tot }} \sim \frac{R_{\text {in }}}{H} \sim 30 \zeta_{-3}^{1 / 2} \chi_{0.1}^{-1}
$$

where $\chi_{0.1}=\chi / 0.1$, and $\zeta_{-3}=\zeta / 10^{-3}$. These blobs can be maintained on a diffusion timescale of $\sim H / r_{\mathrm{L}} c$, where $r_{\mathrm{L}}$ is the Larmor radius (Cheng \& Lu 2001).

When a blob reaches $\hat{r}_{\mathrm{ms}}$ and be dragged into the black hole, a huge amount of energy will be extracted via the BZ process (Blandford \& Znajek 1977), giving birth to a miniburst. This process may repeat many times till all blobs within $\hat{r}_{\text {sp }}$ are removed (c.f. Eq.(9)). As a result, many mini-bursts should be produced. We propose that these mini-bursts add together to form a GRB, and each mini-burst corresponds to a mini-pulse in the GRB light curve.

The timescale of each mini-pulse is determined by $t_{\text {pulse }} \sim \max \left(\triangle t_{\mathrm{p}}, t_{\mathrm{ff}}\right)($ Cheng \& Lu 2001), where $t_{\mathrm{ff}}$ is the free fall time. Assuming that the black hole is accreting at the Eddington rate $(\dot{m}=1)$, we derive the characteristic duration of the mini-pulse as,

$$
t_{\text {pulse }}=t_{\mathrm{ff}}=3 R_{\mathrm{s}} / c \simeq 3 M_{5} \mathrm{~s},
$$

which is mainly determined by the black hole mass.

In our model, the total duration of a GRB is determined by the timescale of the thermal instability, i.e.,

$$
t_{\text {duration }}=\frac{R_{\mathrm{sp}}}{v_{\mathrm{R}}}=50 \alpha^{-1} \hat{r}_{\mathrm{ms}}^{3 / 2} M_{5} \mathrm{~s}
$$

where $v_{\mathrm{R}}$ is the radial velocity of the matter, and $v_{\mathrm{R}}=\alpha \sqrt{G M_{\mathrm{bh}} / R_{\mathrm{sp}}}$ (Shakura \& Sunvaev 1973). In this main burst phase, the majority of matter within the range of $1<\hat{r}<\hat{r}_{\mathrm{sp}}$ will fall into the black hole. It will take a time that equals to the accretion timescale $\left(t_{\text {acc }}\right)$ to replenish this mass. However, the accretion timescale is very long, $t_{\text {acc }} \sim 3.0 \times 10^{6} \alpha^{-1} \mathrm{~s}$ by assuming $\beta=1$ (c.f. Eq.(4)). Within this time, the accretion rate will decrease to a 
value (c.f. Eq.(2)) that is much lower than the critical accretion rate of $\dot{m}_{\mathrm{c}}$. Consequently, the radiation pressure of the disk is too low to produce enough magnetic field to trigger any additional mini-bursts again (Cheng \& Lu 2001).

Assuming that the magnetic field component normal to the black hole horizon equals to the amplified magnetic field $\left(B_{\mathrm{p}}\right)$, we can further estimate the power and energy extracted via the BZ process in a single mini-burst (Cheng \& Lu 2001), respectively

$$
\begin{aligned}
& P_{\text {pulse }}=1.7 \times 10^{48} A^{2} f(A) M_{5}^{2}\left(\frac{B_{\mathrm{p}}}{10^{9} \mathrm{G}}\right)^{2} \hat{r}_{\text {in }}^{-9 / 8} \mathrm{ergs} / \mathrm{s}, \\
& E_{\text {pulse }} \simeq P_{\text {pulse }} \triangle t_{\mathrm{p}} \simeq 2.69 \times 10^{50} A^{2} f(A) \alpha^{-1} M_{5} \hat{r}_{\text {in }}^{-57 / 64} \mathrm{ergs},
\end{aligned}
$$

where $A$ is the dimensionless angular momentum of the black hole, and $f(A)=2 / 3$ for $A \rightarrow 0$ and $f(A)=\pi-2$ for $\mathrm{A} \rightarrow 1$ (Lee et al. 2000). It has been known that some stellar mass black holes in X-ray binaries and supermassive black holes in centers of galaxies may have high values of dimensionless angular momentum (see e.g., Zhang, Cui \& Chen 1997; Liu, Zhang \& Zhang 2007). Highly spinning black holes are most likely spun up by gas accretion processes. However, for intermediate mass black holes, it is currently not clear at all if they should be spinning or not. On the other hand, black holes with masses around $10^{4}$ solar masses are not likely found in accreting binaries. Therefore, if they are also not located in centers of galaxies where gas densities my be high enough for fueling significant accretion, they should not be highly spinning. Here, as a conservative approach we take $A=0.1$, which leads to $A^{2} f(A)=0.02$.

Eq. (12) shows that $E_{\text {pulse }}$ is a function of $\hat{r}_{\text {in }}^{-57 / 64}$. Therefore, the maximum and minimum value for $E_{\text {pulse }}$ will be acquired when the seed fields anchored in the disk at $\hat{r}_{\text {in }}=1$ and $\hat{r}_{\text {in }}=\hat{r}_{\mathrm{sp}}$, respectively. Immediately, we derive the maximum value of $E_{\text {pulse }}$ by setting $\hat{r}_{\text {in }}=1$ in Eq. (12),

$$
E_{\text {pulse } \max }=5.38 \times 10^{48} \alpha^{-1} M_{5} \text { ergs. }
$$

The minimum value of $E_{\text {pulse }}$ can be derived by setting $\hat{r}_{\text {in }}=2.52 \dot{m}$ in Eq. (12),

$$
E_{\text {pulse } \min }=2.36 \times 10^{48} \alpha^{-1} M_{5} \dot{m}^{-57 / 64} \mathrm{ergs} .
$$

The average value of $E_{\text {pulse }}$ for each pulse can be estimated as,

$$
\begin{aligned}
E_{\text {pulse }, \text { ave }} & =\frac{\int_{1}^{\hat{r}_{\mathrm{sp}}} E_{\text {pulse }} d \hat{r}_{\mathrm{in}}}{\int_{1}^{\hat{r}_{\mathrm{sp}}} d \hat{r}_{\mathrm{in}}} \\
& =4.92 \times 10^{49} \alpha^{-1} M_{5} \frac{(2.52 \dot{m})^{7 / 64}-1}{2.52 \dot{m}-1} \mathrm{ergs} .
\end{aligned}
$$




\subsection{Application to GRB 060614}

We suggest that GRB 060614 could be produced by the tidal disruption of a solar type star $\left(m_{*}=1, r_{*}=1\right)$ by an IMBH. Here we give a rough estimate for the parameters involved in this case. As described in Section 2, the first short episode of emission lasting for $\sim 4 \mathrm{~s}$ is actually composed of $\sim 5$ mini-pulses. We notice that the durations of these mini-pulses can be as short as $\sim 0.6 \mathrm{~s}$. According to our Eq. (10), we estimate the mass of the IMBH as $M_{5} \approx 0.2$. Taking a typical value of $\alpha=0.1$, we then calculate the theoretical duration of the GRB as $t_{\text {duration }} \approx 100 \mathrm{~s}$ from Eq. (11). This is in good agreement with the observed duration of $\sim 102$ s.

Assuming that the GRB happens when the IMBH is roughly accreting at the Eddington rate of $\dot{m}=1$, we can further calculate the average pulse energy (see Eq. (15)) as $E_{\text {pulse,ave }} \approx$ $6.88 \times 10^{48} \mathrm{ergs}$. The exact number of mini-pulses is not easy to determine from observations (it depends too strongly on the timing resolution of the light curve.). From our Eq. (9), we assume that there are $N_{\text {tot }} \sim 30$ mini-pulses, then we get the total burst energy as $E_{\text {tot }}=N_{\text {tot }} E_{\text {pulse,ave }} \approx 2.06 \times 10^{50}$ ergs. Assuming a beaming factor of $\sim 100$ (Gehrels et al. 2006), it will correspond to an isotropic kinetic energy of $\sim 2 \times 10^{52} \mathrm{ergs}$, which is consistent with the observed energetics of GRB 060614 (see Section 2).

As noted in Section 2, there is a hint of a $9 s$ periodicity between 7 and $50 \mathrm{~s}$ in the $\gamma$-ray light curve (Gehrels et al. 2006). Such a quasi-periodic oscillation can be naturally explained in our model. We suggest that this periodicity should be connected with the Kepler motion at $R_{\mathrm{ms}}$. In fact, the Kepler period is (Shakura \& Sunvaev 1973)

$$
t_{\mathrm{K}}=2 \pi \sqrt{\frac{R_{\mathrm{ms}}^{3}}{G M_{\mathrm{bh}}}} \approx 50 \hat{r}_{\mathrm{ms}}^{3 / 2} M_{5} \mathrm{~s} .
$$

Taking $M_{5}=0.2$, we find that $t_{\mathrm{K}} \sim 10 \mathrm{~s}$, in good accordance with the observed 9 -s periodicity. It thus seems clear that the falling of the blobs into the black hole is neither uniform nor completely unsystematic. They seem to fall in group and the falling is modulated by the Keplerian motion. However, the detailed modulation mechanism is still largely uncertain and needs further investigations.

Our model can also naturally explain other basic features of GRB 060614. For example, it needs not to be associated with a supernova and need not to reside in an active star forming galaxy. It also needs not to be at the center of its host galaxy, since the black hole involved is not a super-massive one. In our model, the highly collimated outflow can also be naturally launched via the BZ process. 


\section{Event rate}

It is interesting to note that another marginally long burst, GRB 060505, with a duration of $\sim 4 \mathrm{~s}$, was also not accompanied by supernova emission (Fynbo et al. 2006). Thus we currently have two confirmed GRBs that are of our interest. Based on this fact, we can give a rough estimate for the observed event rate of such a phenomenon. Till the end of March 2008, about 600 GRBs have been well localized (Greiner 2008). Optical afterglows have been detected from 233 events of them, with redshifts measured in 141 cases. According to an earlier study by Zeh, Klose, and Hartmann (2004), supernova signature is most likely detectable for a GRB with redshift $z<0.7$, if it is really associated with a supernova. For GRBs with redshifts $z>0.7$, the signature may be too weak to detect. Taking $z<0.7$ as a criterion, we then have a sample of 32 GRBs, among them are GRBs $060505(z=0.089)$ and $060614(z=0.125)$. It hints that about $6 \%$ GRBs are GRB 060614-like. Note that the local GRB-XRF (X-ray flash) birth rate is $\sim(0.5-2) \mathrm{Gpc}^{-1} \mathrm{yr}^{-1}$, or $\sim(0.025-0.1) \mathrm{Myr}^{-1}$ galaxy $^{-1}$ (Schmidt 2001; Zhang \& Mészáros 2004). We then estimate the birth rate of GRB 060614-like event as $\sim(0.03-0.12) \mathrm{Gpc}^{-1} \mathrm{yr}^{-1}$, or $\sim(0.0015-0.006) \mathrm{Myr}^{-1}$ galaxy $^{-1}$. The above calculation is based on the assumption that the GRB emission is isotropic. If we assume a true-to-observed beaming correction factor of $100-1000$, then the local birth rate of GRB 060614-like event will be amplified to $\sim(3-120) \mathrm{Gpc}^{-1} \mathrm{yr}^{-1}$, or $\sim(0.15-$ 6) $\mathrm{Myr}^{-1}$ galaxy ${ }^{-1}$.

In our model, the birth rate of IMBH-induced GRBs in a typical galaxy can be calculated as

$$
\Gamma=\kappa \Gamma_{\mathrm{t}} N_{\mathrm{IMBHs}}
$$

where $N_{\text {IMBHs }}$ is the total number of IMBHs in the galaxy, $\kappa$ is the number ratio of IMBHs whose masses are suitable for producing a GRB, and $\Gamma_{t}$ is the mean tidal disruption rate of a solar type star by an IMBH. We believe that to produce a GRB, the mass of the IMBH should be larger than $\sim 1 \times 10^{4} M_{\odot}$. So, $\kappa$ should be the fraction of IMBHs with mass of $\sim 1 \times 10^{4}-10^{5} M_{\odot}$ among all the IMBHs with mass of $\sim 20-10^{5} M_{\odot}$. However, direct evidence for the existence of IMBHs is still lacking, so the three quantities $\left(\kappa, \Gamma_{\mathrm{t}}, N_{\mathrm{IMBHs}}\right)$ involved in Equation (17) are all largely uncertain. Here we can only give some preliminary discussion.

Although the true existence of IMBHs and their number in a typical galaxy is uncertain (Mapelli et al. 2006), Volonteri et al. (2003) has discussed the density of IMBHs under the assumption that the IMBHs are born in $3-3.5 \sigma$ fluctuations collapsing at a given redshift. Assuming that IMBHs are modeled as a halo population distributed following a Navarro Frenk \& White (NFW) or a more concentrated Diemand, Madau \& Moore (DMM) density profile, Mapelli et al. (2006) derived an upper limit for the density of IMBHs via dedicated 
$N$-body simulation. Based on their work, the number of IMBHs in the Milky Way should be $N_{\text {IMBHs }} \leq 10^{3}-10^{4}$.

The parameter $\kappa$ should seriously depend on the mass function of IMBHs. However, since the formation mechanism of IMBHs and their evolution after birth are completely uncertain, we actually cannot give any robust estimate for $\kappa$. Also, the environment that IMBHs reside in is unknown, so that a reliable estimate for the tidal disruption rate $\left(\Gamma_{t}\right)$ is again impossible.

Although it is still impossible to estimate the rate of producing GRB 060614-like GRBs in our model, we believe that this model provides a viable GRB production mechanism. In the future, when more and more GRB 060614-like events are observed, more stringent constraints on $\kappa, \Gamma_{\mathrm{t}}$ and $N_{\mathrm{IMBHs}}$ may be available, and a valuable new window will be open for the study of IMBHs.

\section{Discussion and conclusion}

We have shown that the tidal disruption of a solar type star by an IMBH with a mass of $2.0 \times 10^{4} M_{\odot}$ can be a possible mechanism for the special event of GRB 060614, which is a nearby long burst but is not associated with a supernova. We argue that the powerful energy extracted via the BZ process is enough to trigger a GRB, when the black hole is accreting at the Eddington rate. The basic observed features of GRB 060614 can all be reasonably explained in our frame work.

The black hole mass considered here is higher for ULXs (Baumgardt et al. 2006) and lower for X-ray flares (Komossa et al. 2004) via tidal capture and disruption events. Therefore, no ULXs or X-ray flares could be observed to be associated with the prompt $\gamma$-ray emission in this case.

A GRB produced by the tidal disruption of a star by an IMBH may also leave some hints in its afterglow. It is interesting to note that an oscillatory phenomenon has been observed in the optical afterglow of GRB 050922C, with a period of $\sim 7.2 \mathrm{~min}$ (Zhilyaev et al. 2007). Zhilyaev et al. (2007) suggested that GRB 050922C may be resulted from tidal disruption of a white dwarf star by an IMBH. The observed periodicity in the afterglow is interpreted as due to the precession of an accretion disc.

We thank the anonymous referee for many useful suggestions that lead to an overall improvement of our manuscript. We also would like to thank K.S. Cheng, B. Zhang, Y. Z. Fan, Z. Zheng, Z. G. Dai and X. D. Li for their helpful comments and discussions. 
This research was supported by the National Natural Science Foundation of China (Grants

10273011, 10573021, 10433010, 10625313, 10521001, 10733010, 10725313 and 10221001), and by Chinese Academy of Science through project No. KJCX2-YW-T03.

\section{REFERENCES}

Abramowicz, M.A., 1985, PASJ, 37, 727

Alexander, T., 2005, Physics Reports, 419, 65

Amati L., Della Valle M., Frontera F., Malesani D., Guidorzi C., Montanari E., Pian E., 2007, A\&A, 463, 913

Ayal, S., Livio, M., \& Piran, T. 2000, ApJ, 545, 772

Baumgardt, H., Hopman, C., Portegies Zwart, S., \& Makino, J., 2006, MNRAS, 372, 467

Blinnikov, S.I., Novikov, I.D., Perevodchikova, T.V., \& Polnarev, A.G., 1984, SvAL, 10, 177

Blandford, R.D., \& Znajek, R.L., 1977, MNRAS, 179, 433

Bloom, J.S., et al., 2006, ApJ, 638, 354

Brassart, M., \& Luminet , J.P., 2007, astro-ph/0707.2476v2

Cannizzo, J. K., Lee, H. M., \& Goodman J. 1990, ApJ, 351, 38

Carter, B., \& Luminet, J.-P., 1983, A\&A, 121, 97

Carter, B., 1992, ApJ, 391, L67

Cheng, K.S., \& Lu, Y., 2001, MNRAS, 320, 235

Della Valle, M., et al., 2006, Nature, 444, 1050

Diemand, J., Madau, P., \& Moore, B., 2005, MNRAS, 364,367 (DMM)

Eichler, D., Livio, M., Piran, T., \& Schramm, D.N., 1989, Nature, 340, 126

Evans, C. R., \& Kochanek, C. S. 1989, ApJ, 346, 13

Fruchter, A., et al., 2006, Nature, 441, 6438

Fynbo J.P.U. et al., 2006, Nature, 444, 1047 
Galama, T.J., 1998, Nature, 395, 670

Gal-Yam, A., et al., 2006, Nature, 444, 1053

Gebhardt, K., Rich, R.M., \& Ho, L.C., 2005, ApJ, 634, 1093

Gehrels, N., et al., 2006, Nature, 444, 1044

Gomboc, A. \& C̆adě̆, A., 2005, ApJ, 625, 278

Greiner, J., 2008, http://www.mpe.mpg.de/ jcg/grb.html

Heger, A., \& Woosley, S.E., 2002, ApJ, 567, 532

Jakobsson, P., \& Fynbo, Johan P.U., 2007, astro-ph/0704.1421

Kobayashi, S., Laguna, P., Phinney, E.S., \& Mészáros, P., 2004, ApJ, 615, 855

Komossa, S., \& Greiner, J., 1999, A\&A, 349, L45

Komossa, S., Halpern, J., Schartel, N., Hasinger, G., Santos-Lleo, M., \& Predehl, P., 2004, ApJ, 603, L17

Kouveliotou, C., et al., 1993, ApJ, 413, L101

Laguna, P., Miller, W.A., Zurek, W.H., Davies, M.B., 1993, ApJ, 410, L83

Lee, H.K., Wijers, R.A.M.J., \& Brown, G.E., 2000, 325, 83

Li, L.X., Narayan, R., \& Menou, K., 2002, ApJ, 576, 753

Liu, Y., Zhang, S. N., Zhang, X. L., 2007, PASJ, 59(1), 185

Lu, Y., Cheng, K.S., \& Huang, Y.F., 2006, ApJ, 641, 288

Luminet, J.P., \& Carter, B., 1986, ApJS, 61

Luminet, J.P., \& Pichon, B., 1989, A\&A, 209, 85

Mangano V., et al., 2007, A\&A, 470, 105

Mapelli, M., Ferrara, A., \& Rea, N., 2006, MNRAS, 368, 1340

Mapelli, M., 2007, ApJ, 376, 1317

Markoff, S., Falcke, H., \& Fender, R. 2001, A\&A, 372, L25 
Menou, K, \& Quataert, E., 2001, ApJ, 562, L137

Miller, M.C., \& Hamilton, D.P., 2002, MNRAS, 330, 232

Milosavljević, M., Merritt, D., \& Ho, Luis C., 2006, ApJ, 652, 120

Navarro, J.F., Frenk, C.S., White, S.D.M., 1996, ApJ, 462, 563 (NFW)

Orosz, J.A., in van der Hucht, K.A., Herrero, A., Esteban, C., eds, proc. IAU Symp. 212, A massive star Odyssey: From main sequence to Supernova. Astron. Sco. Pac., San Francisco, P.365

Pian, E., et al., 2006, Nature, 442, 1011

Portegies Zwart, S. F., Baumgardt, H., Hut, P., Makino, J., McMillan, S. L. W., Nature, 428,724

Sanders, R.H., \& Van Oosterom, W., 1984, A\&A, 131, 267

Rees, M. J. 1988, Nature, 333, 523

Shakura, N.I., \& Sunyaev, R.A., 1973, A\&A, 24, 337

Schmidt, M., 2001, ApJ, 552, 36

Trimble, V., \& Aschwanden, M.J., 2002, PASP, 114, 475

Ulmer, A. 1999, ApJ, 514, 180

Villasenor, J.S., et al., 2005, Nature, 437, 855

Volonteri, M., Haardt, F., \& Madau, P., 2003, ApJ, 582, 559

Woosley, S.E., 1993, ApJ, 405, 273

Zeh, A., Klose, S., Hartmann, D.H., 2004, ApJ, 609, 952

Zhang, S. N., Cui, W., Chen, W. 1997, ApJ, 482, L155

Zhang, B., Mészáros, P., 2004, Int. J. Mod. Phys. A, 19, 2385

Zhang, B., et al., 2007, ApJ, 655, L25

Zhilyaev, B.E., Andreev, M.V., Sergeev, A.V., Petkov, V.B., preprint, http://arXiv.org/abs/0711.0038 